\title{
The expanding role of radiation therapy for thymic malignancies
}

\author{
Jonas Willmann, Andreas Rimner
}

Department of Radiation Oncology, Memorial Sloan Kettering Cancer Center, New York, NY, USA

Contributions: (I) Conception and design: All authors; (II) Administrative support: A Rimner; (III) Provision of study materials or patients: None; (IV) Collection and assembly of data: J Willmann; (V) Data analysis and interpretation: All authors; (VI) Manuscript writing: All authors; (VII) Final approval of manuscript: All authors.

Correspondence to: Andreas Rimner, MD. Department of Radiation Oncology, Memorial Sloan Kettering Cancer Center, 1275 York Avenue, New York, NY 10065, USA. Email: rimnera@mskcc.org.

\begin{abstract}
The role of radiation therapy (RT) in thymic malignancies has long been subject to considerable controversy. The main role for RT is in the setting of adjuvant therapy after surgical tumor resection, especially in advanced or incompletely resected cases. However, recent studies with larger patient numbers and cleaner study populations than previous studies have indicated a potentially clearer than previously assumed benefit after post-operative RT (PORT) even for completely resected patients with earlier stages of thymoma. In marginally resectable patients RT may be used in combination with neoadjuvant chemotherapy to shrink tumors and thereby potentially enable resection. In unresectable patients concurrent or sequential chemotherapy and RT can be employed as the definitive nonsurgical approach. The tendency of thymic tumors to recur in the pleural space highlights the necessity for more effective approaches to identify and treat high risk patients. Experiences in other pleural malignancies may pave the way to novel treatment modalities, for example pleural IMRT. The role of these techniques in thymic malignancies has yet to be determined and is not advisable at the current time outside of a clinical study. As the disease often takes an indolent course with excellent longterm local control (LC) and survival, late toxicities related to radiation of the mediastinum and adjacent organs at risk (OARs) have to be taken into consideration and may jeopardize the benefit patients experience from RT, especially in younger patients with a long-anticipated life expectancy. Radiation techniques, such as intensity modulated RT (IMRT) and proton beam therapy (PBT), have substantially reduced the exposure of OARs to ionizing radiation which is expected to translate into reduced long-term toxicities. Hence, the risk-benefit ratio of RT in early stage thymoma patients may be shifted favorably.
\end{abstract}

Keywords: Thymoma; thymic carcinoma; post-operative radiation therapy (PORT); intensity modulated radiation therapy (IMRT); proton beam therapy (PBT)

Submitted Nov 20, 2017. Accepted for publication Jan 23, 2018.

doi: $10.21037 /$ jtd.2018.01.154

View this article at: http://dx.doi.org/10.21037/jtd.2018.01.154

\section{Introduction}

Thymic malignancies, while rare, are the most common primary tumors of the anterior mediastinum in adults with an incidence of approximately 3.2 per 1,000,000 people (1). Epithelial tumors of the thymus appear in a wide range of histologic subtypes. The three large categories are thymoma as being the most common of them (2); thymic carcinomas (TC), the most aggressive form of thymic epithelial tumors; and the even rarer thymic carcinoids or neuroendocrine tumors of the thymus (NETT).

Thymoma and TC are divided into a number of different histologic subtypes. The World Health Organization (WHO) Histologic Classification System, which has been introduced in 2004 (3) and revised in 2014 (4), describes in detail the different histologic subtypes. Based on the morphologic appearance of the tumor, thymoma of types $\mathrm{A}, \mathrm{AB}, \mathrm{B} 1, \mathrm{~B} 2$, $\mathrm{B} 3$ and TC (previously referred to as thymoma type $\mathrm{C}$ ) are 
Table 1 Comparison between the modified Masaoka staging system (7) and the tumor, nodes, metastasis (TNM) based staging system for thymic epithelial tumors included in the AJCC 8th edition cancer staging manual (11-13)

\begin{tabular}{|c|c|c|}
\hline Descriptors & Modified Masaoka clinical staging of thymoma & 8th edition of the AJCC staging system \\
\hline $\begin{array}{l}\text { Involvement beyond } \\
\text { capsule }\end{array}$ & $\begin{array}{l}\text { IIB - macroscopic invasion into surrounding } \\
\text { fatty tissue or grossly adherent to but not } \\
\text { through mediastinal pleura or pericardium }\end{array}$ & $\mathrm{T} 1 \mathrm{~b}$-involvement of mediastinal pleura \\
\hline \multirow{2}{*}{$\begin{array}{l}\text { Involvement of pleura, } \\
\text { pericardium, great } \\
\text { vessels or neighboring } \\
\text { organs }\end{array}$} & \multirow{2}{*}{$\begin{array}{l}\text { III-macroscopic invasion into neighboring } \\
\text { organs (i.e., pericardium, great vessels, or lung) }\end{array}$} & T2-involvement of pericardium \\
\hline & & $\begin{array}{l}\text { T3-involvement of lung, brachiocephalic vein, superior vena } \\
\text { cava, chest wall, phrenic nerve, chest wall, hilar (extrapericardial) } \\
\text { pulmonary vessels }\end{array}$ \\
\hline \multirow{2}{*}{ Nodal involvement } & \multirow{2}{*}{$\begin{array}{l}\text { IVB-lymphogenous or hematogenous } \\
\text { metastasis }\end{array}$} & $\mathrm{N} 1$-anterior (perithymic) nodes \\
\hline & & $\mathrm{N} 2$ - deep intrathoracic or cervical nodes \\
\hline \multirow[t]{3}{*}{ Metastatic spread } & IVA-pleural or pericardial dissemination & MO-no metastatic disease \\
\hline & $\begin{array}{l}\text { IVB-lymphogenous or hematogenous } \\
\text { metastasis }\end{array}$ & M1a-separate pleural or pericardial nodule(s) \\
\hline & & $\begin{array}{l}\text { M1b-pulmonary intraparenchymal nodule or distant organ } \\
\text { metastasis }\end{array}$ \\
\hline
\end{tabular}

distinguished. Depending on the histologic subtype the clinical presentation and prognosis of thymic malignancies can vary dramatically. While thymoma may take an indolent, non-invasive course, TCs and TNET have a much higher aggressive biology with metastatic potential (5).

For the anatomical staging of thymic malignancies, a number of different systems exist. The Masaoka-Koga system, which was developed by Masaoka and colleagues in 1981 (6), and revised in 1994 (7) by Koga and colleagues has to date been the most commonly used staging system due to its prognostic significance $(8,9)$ and is based on the local micro- and/or macroscopic invasion of the tumor and the presence of lymphogenous or hematogenous spread. The International Thymic Malignancies Interest Group (ITMIG) and the Thymic Domain of the Staging and Prognostic Factors Committee (TD-SPFC) of the International Association for the Study of Lung Cancer (IASLC) in conjunction with AJCC has developed a traditional tumor, nodes, metastasis (TNM) system, as used in many other solid tumors which has been introduced in the $8^{\text {th }}$ version of the AJCC staging system. Especially the T component of the new TNM system has similarities to the Masaoka-Koga system and describes the tumor's extension into the adjacent organs. The TNM system also contains differences from the Masaoka-Koga system. For example, the distinction of whether the tumor is encapsulated or invaded through the capsule into the mediastinal fat or mediastinal pleura has been dismissed, as no clinically significant difference in outcomes could be detected and the difficulty in determining the capsule (10) (Table 1). However, this may also be in part due to a lack of statistical power, uniformity in pathologic and surgical description and availability of this level of granularity in the ITMIG/ IASLC database.

To date, the optimal treatment of thymic malignancies remains based on retrospective studies and populationbased analyses and general oncologic principles. Due to the rarity of the disease, there is a lack of randomized, prospective trials to back evidence-based guidelines.

ITMIG was founded to overcome the disagreements 
and promote international collaboration in clinical and translational research on thymic malignancies. This multidisciplinary society has brought together experts from surgical, medical, radiation oncology, and neurology as clinical disciplines, as well as pathologists, radiologists, and basic researchers and aims to accelerate research on this rare orphan disease. Most importantly the first publications from this group focused on the development of agreed upon guidelines for diagnostic workup, staging, and clinical management $(4,10,13-18)$.

One of these included specific radiation therapy (RT) definitions and uniform reporting guidelines (17), another one the creation of an international database for the investigation of clinical issues important to thymic malignancies that existing population-based registries often lack, including histology, treatment, prognosis, autoimmune disease, and second malignancies (18). Using the ITMIG guidelines and database, recent studies analyzed clearly defined subgroups of thymic malignancy patients, e.g., to assess if post-operative RT (PORT) is associated with a survival benefit in patients with completely resected thymoma stages II and III (19).

It is generally agreed upon that surgical resection is the backbone in the treatment of resectable thymic malignancies, and complete resection can cure earlystaged localized thymomas (stage I) without any additional adjuvant treatment. In more advanced stages of disease (stage II or higher), with aggressive histologic subtypes or incomplete resections, the optimal neoadjuvant or adjuvant approach remains unclear.

Novel techniques, such as IMRT or proton beam RT, may reduce long-term radiation toxicity and thereby favorably shift the risk-benefit ratio of RT. Radiation techniques that have been developed for other thoracic malignancies, such as hemithoracic intensity modulated pleural RT (IMPRINT) might be potential options to prevent intrapleural failures. However, they have not yet been formally tested in this patient population. Moreover, medical treatment options, such as targeted therapy or the use of heated intrapleural chemotherapy, may be combined with surgery and RT in the future for multimodal treatment plans that further improve outcomes.

\section{The role of postoperative RT (PORT)}

\section{Thymoma}

Areas of agreement in the management of thymoma along with some of controversy were shown in a survey amongst members of the European Society of Thoracic Surgeons (ESTS) (20). While there was general consensus concerning the importance of complete resection and a multidisciplinary treatment approach, postoperative treatment for stage II thymoma and stage III thymoma were the most controversial issues. In stage II thymoma $60 \%$ of the surveyed centers consider PORT, while $40 \%$ of the centers stated that they do not use PORT. However, most of the center that administer PORT in stage II thymoma stated, that they raise the indication on an individual basis, depending on the histologic subtype, completeness of resection and subdivision of stage II according to the Masaoka-Koga staging system into stage IIA and IIB.

The disagreement found among the ESTS members echoes the debates and conflicting data in the literature. This might be in part because of the rarity of the disease that makes it hard to conduct prospective trials that could lead to level I evidence. Besides that, the results of some studies on thymoma are inconclusive because heterogeneous cohorts are analyzed. For example, combining stage I patients, that have pretty consistently been shown not to benefit from PORT, with more advanced stages might obscure an effect of PORT (21). Even when combining only stages II and III the heterogeneity of the cohort might make it hard to draw meaningful conclusions (22). When analyzing incompletely resected patients, the fraction of patients that have only microscopically residual disease (R1) in relation to those having macroscopically visible residual disease (R2) might also influence the results. Patients with gross residual disease after surgery might have more advanced tumors or a histologically more aggressive subtype. There might often be certain patterns that lead to more patients with adverse high-risk tumor characteristics or suboptimal surgical management such as incomplete resections being referred for PORT $(23,24)$. If despite such imbalances of unfavorable characteristics, the overall survival (OS) of patients receiving PORT and those who only undergo surgery is similar this might indicate that PORT was effective at reducing the risk of recurrence in patients with incomplete resection to a level that is comparable to those of patients with completely resected thymoma (24). Some population-based databases also lack essential patient characteristics that might influence their outcome. There might be no distinction between thymoma and TC, or the different histologic subtypes of thymoma that might possibly respond differently to RT. The outcome measures reported are of major importance 
as well to ensure comparability between studies and not miss the efficacy of a treatment. For example, reporting OS might be insufficient when analyzing thymoma patients. ITMIG recommends recurrence-free survival (RFS), timeto-progression and OS rates at 5 and 10 years for studying the outcomes of thymic malignancy (14). The National Comprehensive Cancer Center (NCCC) acknowledges the limited data currently available and recommends "considering" PORT in completely resected thymoma with no differentiation between stages II to IV, with a category 2B recommendation. Despite the limitations of many studies on thymoma, trends can be observed that provide a basis for recommendations by stage, especially taking into account the most recent publications that analyze clean and well-defined groups of patients.

In stage I thymoma, excellent local control (LC) rates after surgery have been consistently shown in several studies, thus there is no indication for PORT in this setting (25-27).

For incompletely resected stage II thymoma patients it is intuitive and widespread practice to offer adjuvant RT to prevent local recurrence and improve their survival. With R0 resection many series report recurrence rates of $<10 \%$ with or without adjuvant treatment (28-31). Still, there is increasing evidence for PORT even in completely resected stage II disease. Studies from the Surveillance, Epidemiology, and End Results (SEER) databases showed that the subgroup of patients with stage IIB (macroscopic capsular invasion), but not those with stage IIA (microscopic transcapsular invasion), thymoma had a non-significant trend towards improved OS with the addition of PORT (27). A recent analysis of the National Cancer Database (NCDB) also showed an OS benefit from adjuvant RT for completely resected stage IIB thymoma patients (32). These findings align well with a study of the ITMIG database, which might provide the strongest evidence to date supporting the use of PORT in stage II thymoma even if completely resected (19). The 5- and 10-year overall survival rates for patients having undergone an operation plus PORT versus an operation alone were $97 \%$ and $91 \%$ versus $93 \%$ and $83 \%$, respectively $(\mathrm{P}=0.02)$. Taken together, especially the latest studies, that examined large and well-defined cohort of patients, may provide sufficient power to detect a survival benefit from PORT and support its use in thymoma of stages IIB even in completely resected patients, while for IIA thymoma a benefit from PORT is not entirely clear.

For stage III disease, there is a more apparent benefit from PORT and it appears to be greater than in stage II (19). Increased rates of local failure after surgery alone have been reported in many studies and outcomes improved with PORT. For example, one SEER analysis found a median overall survival rate of 97 months with surgery + PORT, compared to 76 months with surgery alone in patients with stages III-IV disease (27). Another study demonstrated approximately a $10 \%$ benefit in 5 -year cumulative survival for resected stage III thymoma with the addition of PORT to surgical resection, from $65 \%$ to $75 \%$ (33). A survival advantage from PORT for stage III patients has also consistently been described in the recent ITMIG and NCDB publications $(19,32)$. Completely resected stage III thymoma treated with operation plus PORT versus an operation alone had 5- and 10-year OS rates of $92 \%$ and $79 \%$ versus $76 \%$ and $64 \%$, respectively $(\mathrm{P}=0.0005)$ in the ITMIG study (19). Common approaches that have demonstrated high levels of disease control in stage III thymoma include neoadjuvant chemotherapy, followed by surgery, and then adjuvant RT (34-36).

If tolerable, aggressive multimodality treatment including neoadjuvant chemotherapy, surgery and adjuvant RT can be considered in patients with localized stage IVA thymoma to improve their survival, although the benefit seems to be less pronounced than in stage III (36).

The impact of histologic subtypes of thymoma on the benefit from PORT in patients is far less clear. While the most recent ITMIG study suggests that WHO type B1, B2, B3 profit more from PORT (19), two NCDB studies demonstrate a greater benefit for the less aggressive WHO type $\mathrm{A}$ and $\mathrm{AB}(22,32)$. This is somewhat contrary to the generally held belief that the subtypes with a more aggressive disease course are more likely to benefit from additional adjuvant RT. Given the rarity of tumor-specificdeath, excellent long-term survival and higher median age of patients with $\mathrm{WHO}$ type $\mathrm{A}$ and $\mathrm{AB}$ tumors compared to those who have more aggressive WHO types B1, B2, B3 a benefit from PORT might have been obscured by solely observing OS, especially at an early stage of disease $(3,37)$. Still, future studies will have to determine how much histologic thymoma subtype should influence treatment decisions.

\section{Thymic carcinoma and NETT}

A survey amongst ITMIG members to find out about management practices in the treatment of TC showed that among the areas of the largest controversy were the optimal adjuvant treatment for completely resected TC of stages II and III (38). The resulting variability in the treatment of 
TC was also confirmed in a survey of ESTS members (20). Half the participating centers reported to treat TC similarly to thymoma of other histologic subtypes, whereas the remaining half stated that they use a completely different approach. This shows that even though the WHO histology classification and the Masaoka staging system are widely accepted among the institutions, they do not uniformly impact treatment modalities.

To uncover treatment patterns of TC patients that are associated with OS or RFS the ITMIG and the ESTS databases were analyzed (39). Patients receiving PORT had an improved OS compared with those who do not receive RT. In addition, a survival benefit from complete (R0) resection status was found. An analysis of the Japanese Association for Research on the Thymus (JART) database that included TC cases only in stages II and III aimed to assess the impact of PORT (23). PORT only improved RFS but not OS of stages II and III TC patients. RFS might however be the more accurate measurement for a benefit from PORT than OS in this series, as the treatment after the relapse of TC varies widely among institutions that were analyzed. Regardless of stage and resection status, a multimodality treatment approach for TC is recommended, combining neoadjuvant chemotherapy, aggressive surgical resection and PORT (40). The magnitude of the effect from PORT in different stages of disease has yet to be quantified. As outcomes are still suboptimal for TC and distant metastases more common than in thymoma, studies examining novel systemic therapies are needed (36).

Concerning NETT specifically, an analysis of the ITMIG and ESTS databases revealed that RT was part of the treatment in $39.5 \%$ of the TNET patients, the vast majority of these cases being PORT (87\%) (41). Most data on the treatment of NETT are derived from case reports or small case series. The largest study the ITMIG and ESTS databases failed to demonstrate any survival benefit from PORT (41). Once again, complete surgical resection was associated with improved survival. However, given the extremely limited number of NETT cases and the fact that data from almost three decades have been evaluated, the role of RT in this setting is far from clear and should be evaluated in future prospective studies.

\section{Neoadjuvant and definitive RT for locally advanced disease}

Only a minority of thymic malignancies present at a locally advanced stage (III or IVA). However, the difficulty to achieve complete resection in these cases sometimes hampers a treatment approach that is primarily based on surgery. Possible options to improve respectability and outcomes include neoadjuvant induction or definitive RT, chemotherapy or a combination of both. Only $4 \%$ of thymic malignancy patients undergo neoadjuvant treatment (chemotherapy, RT or chemoradiation) prior to surgery, data on the efficacy of RT in this setting are therefore more scare than for adjuvant RT (42).

A cohort of 22 patients with locally advanced thymoma and TC (stage III and IV) has been analyzed prospectively to assess the efficacy of neoadjuvant chemoradiation therapy (CRT) and define specific radiographic criteria to preoperatively detect high risk patients, who might profit most from additional neoadjuvant treatment (43). The exact extent of the disease and the histology subtype (TC or thymoma) might significantly influence a patient's risk for incomplete resection or local recurrence. However, as the Masaoka staging system relies on resection and pathologic findings, it remains challenging to stage patients as locallyadvanced preoperatively. Consistent inclusion criteria for studies on neoadjuvant treatment have therefore been lacking. The authors used computed tomography findings such as large tumor size, irregular borders, heterogeneous appearance, ectopic calcification, obvious great vessel or adjacent organ invasion and/or encirclement, and high $\mathrm{SUV}_{\text {max }}$ on positron emission tomography to determine a subset of high-risk patients, who were believed to be locally advanced (III and IV) or have a more aggressive histologic subtype (TC or thymoma WHO type B3). Only patients that were deemed high-risk by radiographic criteria were included in the study and assessed for the efficacy of neoadjuvant CRT. Postoperatively, the patients' actual pathologic stage and the histologic subtype were determined and compared to the preoperative radiographic findings. The radiographic high-risk criteria were able to identify patients who ultimately had locally advanced tumors and high-risk histologic types and might thus guide decisions for additional preoperative treatment. After neoadjuvant concurrent CRT (CCRT), microscopically complete resection (R0) could be achieved in the vast majority $(77 \%)$ of patients and no patient who underwent a complete resection experienced recurrence. The OS benefit was bigger for patients that could be downstaged compared to those whose stage remained unchanged after induction CRT. The response rate to neoadjuvant CRT was greater in TC than it was in thymoma. Induction CRT might therefore be a viable option to achieve complete resection 
in patients with locally advanced and highly aggressive tumors and thereby potentially improve their survival. The appropriate identification of high-risk patients for induction treatment might be worth further investigation.

In patients with locally advanced thymoma and TC (stages III and IV) who undergo a definitive nonsurgical treatment, CCRT provided the biggest improvement of both tumor response and OS compared to subsequent chemotherapy and RT (SCRT) or RT alone in a retrospective single-institution study (44). The overall response rate after CCRT was $87.7 \%$, compared to $50 \%$ and $43.8 \%$, after SCRT and RT alone, respectively. These findings indicate that even patients with advanced disease for whom surgery is not feasible might benefit from nonsurgical treatment, such as CCRT.

\section{Novel approaches in RT}

The best currently available studies on the impact of RT in thymic malignancies to date are based on the analysis of large, retrospective, multi-institutional databases. Common limitations to such studies are the inability to control for selection bias, variation in institutional standards for RT, variation of diagnostic imaging techniques and staging evaluations, and variation regarding RT field design, doses and applied techniques, all of which make it difficult to compare studies. Moreover, as the data have been acquired over a period of sometimes more than two decades and RT has been rapidly evolving, patients have likely been treated with different RT techniques, ranging from conventional RT to three-dimensional conformal RT (3D-CRT) or modern IMRT (45). The implementation of modern, more conformal RT techniques and on-board imaging techniques might have a significant influence on the safety and precision of RT delivery, as has been shown in other types of cancer (46-48). The impact of IMRT and other novel techniques such as proton beam therapy (PBT), optimal dosing and radiation field design have yet to be accounted for in future prospective studies.

A detailed analysis on failure patterns and radiation treatment fields applied the ITMIG reporting guidelines in 156 thymoma patients in stages II to IV and revealed recurrence in the pleura as the most common pattern of failure, occurring in approximately one quarter of patients (49). The majority of patients with pleural failures had stage IV thymoma at diagnosis and thus was at a higher inherent risk for pleural recurrences. As the only marginal recurrence in the study occurred in a patient treated with
3D-CRT and all but one locoregional failure occurred in the pleura, far outside the radiation field, their findings indicated that the margins applied by the institutions and as suggested by the ITMIG guidelines are appropriate when using even more conformal techniques such as IMRT. However, the fact that patients with pleural recurrence had an unfavorable outcome stresses the need for novel therapies to improve the control of pleural disease.

Furthermore, this study confirmed the meaningful applicability of the ITMIG radiation guidelines. Previous studies have lacked detailed descriptions of field designs, as well as uniform definitions for reporting failure patterns. By applying the ITMIG reporting guidelines the authors also created a benchmark for clinical application of these guidelines and to specifically correlate sites of failure to delivered radiation doses. Detailed study of the failure patterns may also inform a revision of the guidelines if needed to adjust for potential RT field design failures and contouring errors.

An option to prevent or treat pleural recurrence might be highly conformal IMPRINT that has been developed and refined in patients with malignant pleural mesothelioma (MPM). Conventional RT techniques have a high risk of causing severe radiation pneumonitis (RP) when high doses are delivered to the thorax and the lungs are not adequately spared. Novel IMPRINT allows for the safe delivery of high doses up to 50 Gy to the entire pleura in MPM patients, even with bilateral intact lungs after surgical pleurectomy/ decortication (50). However, while IMPRINT may be technically feasible to deliver and might be beneficial in MPM patients, whose prognosis tends to be grim, it remains far from clear whether it will be appropriate in thymoma patients. These patients have a much longer and more favorable prognosis, even in the setting of pleural recurrences. Thus, long-term toxicities that may not have been observed in MPM patients due to their short median survival may occur in thymoma patients and expose them to an undue risk. Whether such an aggressive treatment is safe, feasible and warranted to treat thymoma is thus unclear and in need of further careful investigation. Its use should be limited to carefully selected patients in centers with great experience with this technique and appropriate consenting.

Highly conformal radiation techniques such as IMRT might also favorably influence the outcome of thymoma and TC patients. A retrospective single-institution study found that adjuvant IMRT and 3D-CRT might achieve better outcomes than conventional RT as an adjuvant treatment of completely resected stage III thymoma patients (51). 
Interestingly, the authors found that in the 3D-CRT/IMRT cohort median OS was significantly improved compared to OS in the surgery alone group, while no benefit was detected in the conventional RT cohort. Large radiation fields, poor accuracy and higher normal tissue toxicities in conventional RT compared to 3D-CRT/IMRT might explain these findings. In other thoracic malignancies, such as non-small cell lung cancer (NSCLC) (47) or MPM (48), IMRT and the combination with four-dimensional computed tomography (4DCT) simulation could reduce radiation induced toxicities with improved LC and OS. Still, to ensure optimal target delineation and treatment delivery with these techniques, studies on their use in thymic malignancies are needed.

Besides IMRT with photons, $\mathrm{PBT}$ is a promising technique to reduce the mean doses delivered to the organs at risk (OARs). The characteristic Bragg Peak of proton beams results in a lower entrance dose compared to photon RT and little to no exit dose. PBT therefore holds the potential to further reduce the risk of long-term cardiopulmonary toxicities and secondary malignancies. Excellent survival and LC rates have been shown in NSCLC patients, as well as decreased toxicities compared to photon IMRT $(52,53)$. Until recently, PBT in thymic malignancies has only been described in a very limited number of case reports. For example, the use postoperative PBT was described for a 23-year-old patient with stage III thymoma (WHO type B2/3) and positive margins after surgery (54). PBT was considered because even with highly conformal IMRT, the mean doses to the OARs were rather high. Compared to the photon IMRT plan, the proton beam plan reduced the mean dose to the heart by $8 \mathrm{~Gy}$ (28\%), lungs by $6 \mathrm{~Gy}(37 \%)$, and esophagus by $20 \mathrm{~Gy}(55 \%)$, whereas it increased the dose to the breasts by 2 Gy $(-27 \%)$. The first prospective study recently showed that PBT in thymoma and TC patients is safe and feasible (55). Twentyseven thymoma and TC patients, treated with definitive, salvage or adjuvant double-scattering PBT (DS-PBT) over the course of 4 years, were assessed for early response and toxicities. Toxicities, as assessed weekly during proton therapy, at 1 month and 3 months after completion of RT, and every 3 to 6 months thereafter, were extremely low, with no CTCAE v4.02 grade $\geq 3$ acute and late toxicities. On early follow-up, no in-field recurrences were reported and disease control was excellent. In the end, the benefit of PBT over conventional RT is substantially influenced by the incidence of late toxicities and local failures. Longterm follow-up studies are therefore needed in order to determine, whether PBT can favorably shift the riskbenefit ratio of RT in patients with thymic malignancies. The impact of alternative PBT techniques ought to be determined as well. Pencil beam scanning proton therapy has been shown to spare OARs even better than passive scatter techniques in other malignancies $(56,57)$. Whether the use of pencil beam scanning proton therapy in thymic malignancy patients will be feasible, despite its susceptibility to intrafractional motion, and whether combining PBT with motion management techniques, such as deep-inspiration breath hold may further improve target coverage and reduce doses to OARs still needs to be clarified.

\section{Conclusions}

In recent years, the role of RT in the treatment of thymoma, TC and NETT has been refined. The decision for adjuvant or neoadjuvant treatment should be based on stage of disease, resection status and histology, although some uncertainties remain due to the lack of prospective trials.

For thymoma, evidence increases for the use of PORT depending on stage and resection status. There is no role for PORT in stage I thymoma. Stage IIA patients probably do not benefit from PORT, while stage IIB patients possibly benefit from adjuvant RT, even if resected completely. Locally advanced stages III, IVA and IVB (lymph node disease only) probably benefit even more from additional RT after surgery. Incompletely resected thymoma patients should generally be considered for RT after surgery to improve LC and improve their survival, especially in case of gross residual disease. If and in how far the different histologic subtypes of thymoma should influence therapeutic decisions still remain elusive. A stronger benefit from PORT has been shown the rather aggressive WHO types B1, B2, B3, but there are contradicting and less intuitively reports of a greater magnitude in the more benign $\mathrm{WHO}$ types $\mathrm{A}$ and $\mathrm{AB}$.

TC should be treated with a multimodality approach including radical surgery and PORT. Adjuvant RT is probably beneficial irrespective of surgical margin status. The effect on TC seems to be more pronounced than on thymoma, even though the benefit of PORT in different stages is not clearly defined. A benefit from PORT has not been proven definitively for NETT patients. Still, RT is occasionally applied postoperatively.

Patients with locally advanced thymic malignancies might benefit from neoadjuvant treatment, including RT and possibly chemotherapy, to enable complete resection, 
improve LC and prolong survival. Especially TC patients appear to profit from induction therapy. In combination with chemotherapy, definitive RT might be a viable option for treating patients that are deemed inoperable.

For young patients with early stage thymoma whose cancer-related mortality is low, late cardiopulmonary toxicities and secondary malignancies from the exposure to radiation are of serious concern. The advantages of novel techniques are expected to favorably shift the riskbenefit ratio. Studies on highly conformal IMRT or PBT for treating thymic malignancies are scarce. Still, there are some promising data, suggesting favorable toxicity profiles and improved outcomes.

The effort to create standardized reporting definitions by ITMIG and other large international focus groups has helped generating well-powered retrospective studies that analyze large-scale databases on thymic malignancies. Prospective trials, using standardized and comparable outcome measures and reporting guidelines are preferable to clarify the role of RT in the treatment of different malignancies of the thymus and settle the existing dispute.

\section{Acknowledgements}

None.

\section{Footnote}

Conflicts of Interest: The authors have no conflicts of interest to declare.

\section{References}

1. de Jong WK, Blaauwgeers JL, Schaapveld M, et al. Thymic epithelial tumours: A population-based study of the incidence, diagnostic procedures and therapy. Eur J Cancer 2008;44:123-30.

2. Engels EA. Epidemiology of Thymoma and Associated Malignancies. J Thorac Oncol 2010;5:S260-5.

3. Kondo K, Yoshizawa K, Tsuyuguchi M, et al. WHO histologic classification is a prognostic indicator in thymoma. Ann Thorac Surg 2004;77:1183-8.

4. Marx A, Ströbel P, Badve SS, et al. ITMIG Consensus Statement on the Use of the WHO Histological Classification of Thymoma and Thymic Carcinoma: Refined Definitions, Histological Criteria, and Reporting. J Thorac Oncol 2014;9:596-611.

5. Suster S, Rosai J. Thymic carcinoma. A clinicopathologic study of 60 cases. Cancer 1991;67:1025-32.

6. Masaoka A, Monden Y, Nakahara K, et al. Follow-up study of thymomas with special reference to their clinical stages. Cancer 1981;48:2485-92.

7. Koga K, Matsuno Y, Noguchi M, et al. A review of 79 thymomas: Modification of staging system and reappraisal of conventional division into invasive and non-invasive thymoma. Pathol Int 1994;44:359-67.

8. Hosaka Y, Tsuchida M, Toyabe S. Masaoka Stage and Histologic Grade Predict Prognosis in Patients With Thymic Carcinoma. Ann Thorac Surg 2010;89:912-7.

9. Masaoka A. Staging System of Thymoma. J Thorac Oncol 2010;5:S304-12.

10. Detterbeck FC, Stratton K, Giroux D, et al. The IASLC/ ITMIG Thymic Epithelial Tumors Staging Project: Proposal for an Evidence-Based Stage Classification System for the Forthcoming (8th) Edition of the TNM Classification of Malignant Tumors. J Thorac Oncol 2014;9:S65-72.

11. Nicholson AG, Detterbeck FC, Marino M, et al. The IASLC/ITMIG Thymic Epithelial Tumors Staging Project: Proposals for the T component for the Forthcoming (8th) Edition of the TNM Classification of Malignant Tumors. J Thorac Oncol 2014;9:S73-80.

12. Kondo K, Van Schil P, Detterbeck FC, et al. The IASLC/ITMIG Thymic Epithelial Tumors Staging Project: Proposals for the $\mathrm{N}$ and $\mathrm{M}$ Components for the Forthcoming (8th) Edition of the TNM Classification of Malignant Tumors. J Thorac Oncol 2014;9:S81-7.

13. Detterbeck FC, Asamura H, Crowley J, et al. The IASLC/ITMIG Thymic Malignancies Staging Project: Development of a Stage Classification for Thymic Malignancies. J Thorac Oncol 2013;8:1467-73.

14. Huang J, Detterbeck FC, Wang Z, et al. Standard Outcome Measures for Thymic Malignancies. J Thorac Oncol 2011;6:S1691-7.

15. Detterbeck FC, Moran C, Huang J, et al. Which Way is Up? Policies and Procedures for Surgeons and Pathologists Regarding Resection Specimens of Thymic Malignancy. J Thorac Oncol 2011;6:S1730-8.

16. Benveniste MF, Korst RJ, Rajan A, et al. A Practical Guide from the International Thymic Malignancy Interest Group (ITMIG) Regarding the Radiographic Assessment of Treatment Response of Thymic Epithelial Tumors Using Modified RECIST Criteria. J Thorac Oncol 2014;9:S119-24.

17. Gomez D, Komaki R, Yu J, et al. Radiation Therapy Definitions and Reporting Guidelines for Thymic 
Malignancies. J Thorac Oncol 2011;6:S1743-8.

18. Huang J, Ahmad U, Antonicelli A, et al. Development of the International Thymic Malignancy Interest Group International Database: An Unprecedented Resource for the Study of a Rare Group of Tumors. J Thorac Oncol 2014;9:1573-8.

19. Rimner A, Yao X, Huang J, et al. Postoperative radiation therapy is associated with longer overall survival in completely resected stage II and III thymoma-an analysis of the international thymic malignancies interest group retrospective database. J Thorac Oncol 2016;11:1785-92.

20. Ruffini E, Van Raemdonck D, Detterbeck F, et al. Management of thymic tumors: a survey of current practice among members of the European Society of Thoracic Surgeons. J Thorac Oncol 2011;6:614-23.

21. Liu Q, Gu Z, Yang F, et al. The role of postoperative radiotherapy for stage I/II/III thymic tumor-Results of the ChART retrospective database. J Thorac Dis 2016;8:687-95.

22. Boothe D, Orton A, Thorpe C, et al. Postoperative Radiotherapy in Locally Invasive Malignancies of the Thymus: Patterns of Care and Survival. J Thorac Oncol 2016;11:2218-26.

23. Omasa M, Date H, Sozu T, et al. Postoperative radiotherapy is effective for thymic carcinoma but not for thymoma in stage II and III thymic epithelial tumors: The Japanese Association for Research on the Thymus Database Study. Cancer 2015;121:1008-16.

24. Rimner A. Postoperative radiotherapy: Not all thymic malignancies are created equal. Cancer 2015;121:972-4.

25. Park HS, Shin DM, Lee JS, et al. Thymoma. A retrospective study of 87 cases. Cancer 1994;73:2491-8.

26. Forquer JA, Rong N, Fakiris AJ, et al. Postoperative Radiotherapy After Surgical Resection of Thymoma: Differing Roles in Localized and Regional Disease. Int J Radiat Oncol Biol Phys 2010;76:440-5.

27. Fernandes AT, Shinohara ET, Guo M, et al. The Role of Radiation Therapy in Malignant Thymoma: A Surveillance, Epidemiology, and End Results Database Analysis. J Thorac Oncol 2010;5:1454-60.

28. Singhal S, Shrager JB, Rosenthal DI, et al. Comparison of stages I-II thymoma treated by complete resection with or without adjuvant radiation. Ann Thorac Surg 2003;76:1635-41; discussion 1641-2.

29. Rena O, Papalia E, Oliaro A, et al. Does adjuvant radiation therapy improve disease-free survival in completely resected Masaoka stage II thymoma? Eur J Cardiothorac Surg 2007;31:109-13.
30. Berman AT, Litzky L, Livolsi V, et al. Adjuvant radiotherapy for completely resected stage 2 thymoma. Cancer 2011;117:3502-8.

31. Utsumi T, Shiono H, Kadota Y, et al. Postoperative radiation therapy after complete resection of thymoma has little impact on survival. Cancer 2009;115:5413-20.

32. Jackson MW, Palma DA, Camidge DR, et al. The Impact of Postoperative Radiotherapy for Thymoma and Thymic Carcinoma. J Thorac Oncol 2017;12:734-44.

33. Weksler B, Shende M, Nason KS, et al. The role of adjuvant radiation therapy for resected stage III thymoma: a population-based study. Ann Thorac Surg 2012;93:18228; discussion 1828-9.

34. Kim ES, Putnam JB, Komaki R, et al. Phase II study of a multidisciplinary approach with induction chemotherapy, followed by surgical resection, radiation therapy, and consolidation chemotherapy for unresectable malignant thymomas: final report. Lung Cancer 2004;44:369-79.

35. Huang J, Riely GJ, Rosenzweig KE, et al. Multimodality Therapy for Locally Advanced Thymomas: State of the Art or Investigational Therapy? Ann Thorac Surg 2008;85:365-7.

36. Modh A, Rimner A, Allen PK, et al. Treatment Modalities and Outcomes in Patients With Advanced Invasive Thymoma or Thymic Carcinoma. Am J Clin Oncol 2016;39:120-5.

37. Okumura M, Ohta M, Tateyama H, et al. The World Health Organization histologic classification system reflects the oncologic behavior of thymoma. Cancer 2002;94:624-32.

38. Shepherd A, Riely G, Detterbeck F, et al. Thymic Carcinoma Management Patterns among International Thymic Malignancy Interest Group (ITMIG) Physicians with Consensus from the Thymic Carcinoma Working Group. J Thorac Oncol 2017;12:745-51.

39. Ahmad U, Yao X, Detterbeck F, et al. Thymic carcinoma outcomes and prognosis: results of an international analysis. J Thorac Cardiovasc Surg 2015;149:95-100, 101. e1-2.

40. Spaggiari L, Casiraghi M, Guarize J. Multidisciplinary treatment of malignant thymoma. Curr Opin Oncol 2012;24:117-22.

41. Filosso PL, Yao X, Ahmad U, et al. Outcome of primary neuroendocrine tumors of the thymus: A joint analysis of the International Thymic Malignancy Interest Group and the European Society of Thoracic Surgeons databases. J Thorac Cardiovasc Surg 2015;149:103-9.e2.

42. Wei Y, Gu Z, Shen Y, et al. Preoperative induction therapy 
for locally advanced thymic tumors : a retrospective analysis using the ChART database. J Thorac Dis 2016;8:665-72.

43. Korst RJ, Bezjak A, Blackmon S, et al. Neoadjuvant chemoradiotherapy for locally advanced thymic tumors: A phase II, multi-institutional clinical trial. J Thorac Cardiovasc Surg 2014;147:36-44, 46.e1.

44. Wang CL, Gao LT, Lv CX, et al. Outcome of nonsurgical treatment for locally advanced thymic tumors. J Thorac Dis 2016;8:705-10.

45. Gomez D, Komaki R. Technical advances of radiation therapy for thymic malignancies. J Thorac Oncol 2010;5:S336-43.

46. Grills IS, Yan D, Martinez AA, et al. Potential for reduced toxicity and dose escalation in the treatment of inoperable non-small-cell lung cancer: A comparison of intensitymodulated radiation therapy (IMRT), 3D conformal radiation, and elective nodal irradiation. I Int J Radiat Oncol Biol Phys 2003;57:875-90.

47. Liao ZX, Komaki RR, Thames HD, et al. Influence of Technologic Advances on Outcomes in Patients With Unresectable, Locally Advanced Non-Small-Cell Lung Cancer Receiving Concomitant Chemoradiotherapy. Int J Radiat Oncol Biol Phys 2010;76:775-81.

48. Shaikh F, Zauderer MG, von Reibnitz D, et al. Improved Outcomes with Modern Lung-Sparing Trimodality Therapy in Patients with Malignant Pleural Mesothelioma. J Thorac Oncol 2017;12:993-1000.

49. Rimner A, Gomez DR, Wu AJ, et al. Failure Patterns Relative to Radiation Treatment Fields for Stage II-IV Thymoma. J Thorac Oncol 2014;9:403-9.

50. Rimner A, Zauderer MG, Gomez DR, et al. Phase II study of hemithoracic intensity-modulated pleural radiation

Cite this article as: Willmann J, Rimner A. The expanding role of radiation therapy for thymic malignancies. J Thorac Dis 2018;10(Suppl 21):S2555-S2564. doi: 10.21037/jtd.2018.01.154 therapy (IMPRINT) as part of lung-sparing multimodality therapy in patients with malignant pleural mesothelioma. J Clin Oncol 2016;34:2761-8.

51. Fan C, Feng Q, Chen Y, et al. Postoperative radiotherapy for completely resected Masaoka stage III thymoma: a retrospective study of 65 cases from a single institution. Radiat Oncol 2013;8:199.

52. Kesarwala AH, Ko CJ, Ning H, et al. Intensity-Modulated Proton Therapy for Elective Nodal Irradiation and Involved-Field Radiation in the Definitive Treatment of Locally Advanced Non-Small Cell Lung Cancer: A Dosimetric Study. Clin Lung Cancer 2015;16:237-44.

53. Nguyen QN, Ly NB, Komaki R, et al. Long-Term Outcomes after Proton Therapy, with Concurrent Chemotherapy, for Stage II-III Inoperable Non-Small Cell Lung Cancer. Radiother Oncol 2015;115:367-72.

54. Figura N, Hoppe BS, Flampouri S, et al. Postoperative proton therapy in the management of stage III thymoma. J Thorac Oncol 2013;8:e38-40.

55. Vogel J, Berman AT, Lin L, et al. Prospective study of proton beam radiation therapy for adjuvant and definitive treatment of thymoma and thymic carcinoma : Early response and toxicity assessment. Radiother Oncol 2016;118:504-9.

56. Swisher-McClure S, Teo BK, Kirk M, et al. Comparison of Pencil Beam Scanning Proton- and Photon-Based Techniques for Carcinoma of the Parotid. Int J Part Ther 2016;2:526-32.

57. Ojerholm E, Kirk ML, Thompson RF, et al. Pencil-beam scanning proton therapy for anal cancer: a dosimetric comparison with intensity-modulated radiotherapy. Acta Oncol 2015;54:1209-17. 